\title{
ON LINEAR EQUATIONS IN HILBERT SPACE
}

\author{
L. W. COHEN
}

Given an infinite matrix $A=\left\|a_{i j}\right\|$ where $a_{i j}$ is complex and

$$
\sum_{j=1}^{\infty}\left|a_{i j}\right|^{2}<+\infty, \quad i=1,2, \cdots,
$$

the problem of solving the system of linear equations

$$
y_{i}=\sum_{j=1}^{\infty} a_{i j} x_{j}, \quad i=1,2, \cdots,
$$

has been studied from several points of view. For arbitrary $y_{i}$, E. Schmidt ${ }^{1}$ has given necessary and sufficient conditions on the $a_{i j}$, $y_{i}$ so that the system (2) have a solution $x=\left(x_{j}\right) \in H_{2}$ (Hilbert space). Schmidt shows that if a solution exists, the solution of minimum norm is unique, and gives explicit formulas for this solution. If $A$ defines a linear transformation $T$ on $H_{2}$ to $H_{2}, \mathrm{~F}$. Riesz ${ }^{2}$ gives necessary and sufficient conditions that an inverse $T^{-1}$ exist, that is, that the solution $x=T^{-1}(y)$ where $T^{-1}$ is a linear transformation. The following problem stands between these two: Find conditions on the elements of $A$ so that the system (2) have a solution $x \in H_{2}$ for each $y \in H_{2}$. Such conditions will permit the use of Schmidt's formulas to express the minimal solution $x$ for each $y$ but this of course does not imply the existence of an inverse of the matrix $A$. We give a solution of this problem by a method which depends on a property, which seems new, of the $m$-rowed minors of the matrices $A_{i_{1} \cdots i_{m}}=\left\|a_{i_{s}}\right\|_{1 \unlhd_{\diamond} \leqq m ; j \geqq 1}$ and on Cramer's rule.

Let

$$
a\left(i_{1}, \cdots, i_{m} ; j_{1}, \cdots, j_{m}\right)=\operatorname{det}\left\|a_{i_{s} j_{t}}\right\|_{1 \leqq s, t \leqq m}
$$

be the determinant of the columns $j_{1}, \cdots, j_{m}$ of $A_{i_{1}} \cdots i_{m}$. If $B=\left\|b_{i j}\right\|$ satisfies (1) and $B_{i_{1}}^{\prime} \ldots i_{m}$ is the transposed of $B_{i_{1}} \ldots i_{m}$, the determinant $\operatorname{det} A_{i_{1} \cdots i_{m}} B_{i_{1} \cdots i_{m}}^{\prime}=\operatorname{det}\left\|\sum_{k=1}^{\infty} a_{i_{k}} b_{i_{t} k}\right\|_{1 \leqq s, t \leq m}$ is finite. Because of the continuity of a determinant as a function of its elements

$$
\operatorname{det} A_{i_{1} \cdots i_{m}} B_{i_{1} \cdots i_{m}}^{\prime}=\lim _{n} \operatorname{det}\left\|\sum_{k=1}^{n} a_{i_{s} k} b_{i_{t} k}\right\|_{1 \leqq s, t \leqq m} .
$$

Presented to the Society April 23, 1943; received by the editors March 24, 1943.

1 E. Schmidt, Über die Auflösung linearer Gleichungen mit unendlich vielen Unbekannten, Rend. Circ. Mat. Palermo vol. 25 (1908) pp. 53-77. p. 86.

2 F. Riesz, Les systèmes d'equations linéaires a une infinitê d'inconnues, Paris, 1913, 
There is a theorem ${ }^{8}$ on the minors of products of square matrices which, with slight modification in its proof, yields the identity

$$
\begin{aligned}
& \operatorname{det}\left\|\sum_{k=1}^{n} a_{i_{\mathrm{s}} k} b_{i_{t} k}\right\|_{1 \leq s, t \leqq m} \\
&= \sum_{\left[j_{1} \cdots j_{m}\right]}^{n} a\left(i_{1}, \cdots, i_{m} ; j_{1}, \cdots, j_{m}\right) b\left(i_{1}, \cdots, i_{m} ; j_{1}, \cdots, j_{m}\right), \\
& n \geqq m,
\end{aligned}
$$

where the sum is extended over all combinations $j_{1}, \cdots, j_{m}$ in $1, \cdots, n$.

Theorem 1. If $A, B$ satisfy (1), then

$$
\begin{aligned}
\operatorname{det} A_{i_{1} \cdots i_{m}} B_{i_{1}}^{\prime} \cdots i_{m} \\
\quad=\sum_{\left[j_{1} \cdots j_{m}\right]}^{\infty} a\left(i_{1}, \cdots, i_{m} ; j_{1}, \cdots, j_{m}\right) b\left(i_{1}, \cdots, i_{m} ; j_{1}, \cdots, j_{m}\right),
\end{aligned}
$$

where the sum is extended over all combinations of positive integers $j_{1}, \cdots, j_{m}$. The series converges absolutely and

$\left|\operatorname{det} A_{i_{1} \cdots i_{m}} B_{i_{1} \cdots i_{m}}^{\prime}\right| \leqq\left[\operatorname{det} A_{i_{1} \cdots i_{m}} \bar{A}_{i_{1} \cdots i_{m}}^{\prime}\right]^{1 / 2}\left[\operatorname{det} B_{i_{1}} \cdots i_{m} \bar{B}_{i_{1}}^{\prime} \cdots i_{m}\right]^{1 / 2}$.

Proof. By Schwartz' inequality we have, from (4) and (3) with $B=\bar{A}$,

$$
\begin{aligned}
& \sum_{\left\{j_{1}, \cdots j_{m}\right]}^{n}\left|a\left(i_{1}, \cdots, i_{m} ; j_{1}, \cdots, j_{m}\right) b\left(i_{1}, \cdots, i_{m} ; j_{1}, \cdots, j_{m}\right)\right| \\
& \leqq\left[\sum_{\left[j_{1}, j_{m}\right]}^{n}\left|a\left(i_{1}, \cdots, i_{m} ; j_{1}, \cdots, j_{m}\right)\right|^{2}\right]^{1 / 2} \\
& \cdot\left[\sum_{\left[j_{1}, j_{m}\right]}^{n}\left|b\left(i_{1}, \cdots, i_{m} ; j_{1}, \cdots, j_{m}\right)\right|^{2}\right]^{1 / 2} \\
& \leqq\left[\operatorname{det} A_{i_{1} \cdots i_{m}} \bar{A}_{i_{1} \cdots i_{m}}^{\prime}\right]^{1 / 2}\left[\operatorname{det} B_{i_{1} \cdots i_{m}} \bar{B}_{i_{1} \cdots i_{m}}^{\prime}\right]^{1 / 2} \text {, } \\
& n \geqq m \text {. }
\end{aligned}
$$

The conclusion is now evident as a consequence of (3).

If we define

$$
\begin{aligned}
\left|A B^{\prime}\right|=\lim \sup _{m} \sup _{i_{1} \cdots i_{m}} \mid \sum_{\left[j_{1} \cdots j_{m}\right]}^{\infty} a\left(i_{1}, \cdots\right. & \left., i_{m} ; j_{1}, \cdots, j_{m}\right) x \\
& \times b\left(i_{1}, \cdots, i_{m} ; j_{1}, \cdots, j_{m}\right) \mid
\end{aligned}
$$

${ }^{3}$ C. C. MacDuffee, An introduction to abstract algebra, New York, 1940, Theorem 99.1, p. 216. 
we have a Schwarz inequality for matrices:

$$
\left|A B^{\prime}\right| \leqq\left|A \bar{A}^{\prime}\right| 1 / 2\left|B \bar{B}^{\prime}\right|^{1 / 2} \text {. }
$$

The following lemma contains the Gram condition for linear dependence.

LEMMA 1. The following statements are equivalent:

(a) The rows of $A_{1} \ldots m$ are linearly dependent.

(b) Det $A_{1} \ldots m \bar{A}_{1}^{\prime} \ldots m=0$.

(c) All m-rowed minors of $A_{1} \ldots m$ equal zero.

Proof. That (a) implies (c) is immediate. The equivalence of (b) and (c) follows from Theorem 1 with $A=B$. It remains to show that (c) implies (a). This is evident if $m=1$. Assuming this statement for $m-1$, it is true for $m$ if all the $(m-1)$-rowed minors of $A_{m-1}$ vanish. If one such minor does not vanish, say

$$
\operatorname{det}\left\|a_{i j}\right\|_{1 \leqq i, j \leqq m-1} \neq 0,
$$

we denote by $c_{k}$ the cofactor of $a_{k m}$ in the determinant of the first $m$ columns of $A_{1} \ldots m$. Then $c_{m} \neq 0$ and

$$
\sum_{k=1}^{m} c_{k} a_{k j}=0, \quad j=1, \cdots, m-1 .
$$

But this sum vanishes for all other values of $j$ because of (c). Hence (c) implies (a).

Theorem 2. If $A$ satisfies (1), the finite system

$$
y_{i}=\sum_{j=1}^{\infty} a_{i j} x_{j}, \quad i=1, \cdots, m,
$$

has a solution $x \in H_{z}$ for each $y_{1}, \cdots, y_{m}$ if and only if

$$
\operatorname{det} A_{1 \cdots m} \bar{A}_{1}^{\prime} \ldots m \neq 0 \text {. }
$$

Proof. The necessity is a consequence of Lemma 1. If the condition is satisfied, then there is a nonvanishing $a\left(1, \cdots, m ; j_{1}, \cdots, j_{m}\right)$ by (c) of Lemma 1 and a solution $x=\left(x_{j}\right)$ where $x_{j}=0$ for $j \neq j_{1}, \cdots, j_{m}$ and $x_{j_{1}}, \cdots, x_{j_{m}}$ are determined by Cramer's rule.

COROLlary. If $A$ satisfies (1) and the system (2) has a solution $x \in H_{2}$ for each $y \in H_{2}$, then $\operatorname{det} A_{1} \ldots m \bar{A}_{1}^{\prime} \ldots m \neq 0$ for all $m$.

An estimate of the minimum norm of the solution of the finite system (5) may be given in terms of a series of finite minors in $A_{i_{1}} \cdots i_{m}$. 
Let $J=\left[j_{1}, \cdots, j_{m}\right]$ be a combination of $m$ distinct positive integers and let $S_{m}$ be a set of $J$ such that no two $J$ 's have a common integer while every positive integer is in some $J \in S_{m}$. Let

$$
\begin{aligned}
\alpha_{i_{1}} \cdots i_{m} & =\left[\sup _{S_{m}} \sum_{J \in S_{m}} \mid a\left(i_{1}, \cdots, i_{m} ; j_{1}, \cdots,\left.j_{m}\right|^{2}\right]^{1 / 2},\right. \\
a_{i_{1} \cdots i_{m}} & =\left[\sum_{\left[j_{1} \cdots j_{m}\right]} \mid a\left(i_{1}, \cdots, i_{m} ; j_{1}, \cdots,\left.j_{m}\right|^{2}\right]^{1 / 2} .\right.
\end{aligned}
$$

Since $S_{m}$ is a subset of the sum of all $j_{1}, \cdots, j_{m}$ we have the following lemma.

LEMMA 2. $\alpha_{i_{1}} \ldots i_{m} \leqq a_{i_{1}} \ldots i_{m}$,

$$
\begin{array}{r}
\sup _{S_{m}} \sum_{J \in S_{m}} \sum_{s=1}^{m}\left|a\left(1, \cdots, k-1, k+1, \cdots, m ; j_{1}, \cdots, j_{s-1} j_{s+1}, \cdots, j_{m}\right)\right|^{2} \\
\leqq \\
\sum_{\left[j_{1} \cdots j_{m-1}\right]}^{\infty}\left|a\left(1, \cdots, k-1, k+1, \cdots, m ; j_{1}, \cdots, j_{m-1}\right)\right|^{2} .
\end{array}
$$

THEOREM 3. If A satisfies (1) and the finite system (5) has a solution $x^{m} \in H_{2}$ for each $y^{m}=\left(y_{1}, \cdots, y_{m}, 0,0, \cdots\right)$, then

$$
\inf \left\|x^{m}\right\| \leqq\left\|y^{m}\right\|\left[\sum_{k=1}^{m}\left|\frac{a_{1}, \cdots, k-1, k+1, \cdots, m}{\alpha_{1} \cdots m}\right|^{2}\right]^{1 / 2} .
$$

Proof. By Theorem 2, $\operatorname{det} A_{1} \ldots m \bar{A}_{1}^{\prime} \ldots m \neq 0$ and so $\alpha_{1} \ldots m \neq 0$. Let $M_{k j_{0}}^{m}$ be the cofactor of $a_{k j_{s}}$ in $a\left(1, \cdots, m ; j_{1}, \cdots, j_{m}\right)$. The system (5) has a solution $x_{j_{1}}^{m} \ldots j_{m}$ defined by

$$
x_{x_{j} ; j_{1} \cdots j_{m}}^{m}= \begin{cases}\sum_{k=1}^{m} y_{k} M_{k i}^{m} / a\left(1, \cdots, m ; j_{1}, \cdots, j_{m}\right), & j=j_{1}, \cdots, j_{m}, \\ 0, & j \neq j_{1}, \cdots, j_{m} .\end{cases}
$$

We have

$$
\begin{aligned}
& \left\|x_{j_{1}}^{m} \cdots j_{m}\right\|^{2}\left|a\left(i_{1}, \cdots, i_{m} ; j_{1} \cdots j_{m}\right)\right|^{2} \\
& \leqq \sum_{s=1}^{m}\left|\sum_{k=1}^{m} y_{k} M_{k j_{s}}^{m}\right|^{2} \leqq\left\|y^{m}\right\|^{2} \sum_{s=1}^{m} \sum_{k=1}^{m}\left|M_{k j_{s}}^{m}\right|^{2} \\
& =\left\|y^{m}\right\|^{2} \sum_{s=1}^{m} \sum_{k=1}^{m} \mid a(1, \cdots, k-1, k+1, \cdots, m ; \\
& \left.\quad j_{1}, \cdots, j_{s-1}, j_{s+1}, \cdots, j_{m}\right)\left.\right|^{2} .
\end{aligned}
$$

\section{Hence}




$$
\begin{aligned}
\inf \left\|x^{m}\right\|^{2} \alpha_{1}^{2} \cdots m & \leqq\left\|y^{m}\right\|^{2} \sum_{k=1}^{m} \sup _{S_{m}} \sum_{J \in S_{m}} \sum_{s=1}^{m} \mid a(1, \cdots, k-1, \\
& \leqq\left\|y^{m}\right\|^{2} \sum_{k=1}^{m} a_{1, \cdots, k-1, k+1, \cdots, m}^{2}
\end{aligned}
$$

by Lemma 2. The conclusion follows at once.

A sufficient condition for the solution of the system (2) for each $y \in H$ may be obtained by restricting the constants

$$
\alpha_{m}=\left[\sum_{k=1}^{m}\left|\frac{a_{1} \cdots k-1, k+1, \cdots, m}{\alpha_{1} \ldots m}\right|^{2}\right]^{1 / 2}, \quad m=1,2, \cdots .
$$

TheOREM 4. If $A$ satisfies (1), its rows are linearly independent, and $\alpha=\lim \inf _{m} \alpha_{m}<+\infty$, then for each $y \in H_{2}$ the system (2) has a solution $x \in H_{2}$ such that

$$
\|x\| \leqq \alpha\|y\| \text {. }
$$

Proof. Consider any $y \in H_{2}$ and any $\epsilon>0$. The sequence contains a subsequence $\alpha_{m_{\mu}}<\alpha+\epsilon$. From Theorem 3 it follows that for each $\mu$ there is an $x^{\mu}=\left(x_{j}^{\mu}\right) \in H_{2}$ such that

$$
\begin{aligned}
y_{i} & =\sum_{j=1}^{\infty} a_{i j} x_{j}^{\mu}, \quad i=1, \cdots, m_{\mu}, \\
\left\|x^{\mu}\right\| & \leqq(\alpha+\epsilon)\|y\| .
\end{aligned}
$$

Applying a diagonal process to $\left(x_{j}^{\mu}\right)$ one finds a subsequence $x^{\mu_{\nu}}=\left(x_{j}^{\mu_{\nu}}\right)$ and an $x=\left(x_{j}\right) \in H_{2}$ such that

$$
\begin{aligned}
& \lim _{\nu} x_{j}^{\mu_{\nu}}=x_{j}, \\
& \|x\| \leqq(\alpha+\epsilon)\|y\| .
\end{aligned}
$$

Since for all $\nu, N>0$ and $1 \leqq i \leqq m_{\mu_{\nu}}$

$$
y_{i}-\sum_{j=1}^{\infty} a_{i j} x_{j}=\sum_{j=1}^{N} a_{i j}\left(x_{j}^{\mu_{\nu}}-x_{j}\right)+\sum_{j=N+1}^{\infty} a_{i j} x_{j}^{\mu_{\nu}}-\sum_{j=N+1}^{\infty} a_{i j} x_{j},
$$

$x$ solves the system (1).

Now consider $\epsilon_{n} \downarrow 0$. For each $n$ there is an $x^{n} \in H_{2}$ which solves the system (2) and such that $\left\|x^{n}\right\| \leqq\left(\alpha+\epsilon_{n}\right)\|y\|$. Repeating the diagonal process and the above argument, one finds an $x \in H_{2}$ such that $\|x\| \leqq \alpha\|y\|$ and which solves the system (2).

UNIVERSITY OF WISCONSIN 